\title{
Characterization of High Energy Xe Ion Irradiation Effects in Single Crystal Molybdenum with Depth- resolved Synchrotron Microbeam Diffraction
}

\author{
Di Yun ${ }^{1,2, *}$, Yinbin Miao ${ }^{3}$, Ruqing $\mathrm{Xu}^{1}$, Zhigang $\mathrm{Mei}^{1}$, Kun Mo ${ }^{1}$, Walid Mohamed ${ }^{1}$, Bei Ye ${ }^{1}$, Michael J. Pellin ${ }^{1}$, Abdellatif \\ M. Yacout ${ }^{1}$ \\ ${ }^{1}$ Argonne National Laboratory \\ 9700 South Cass Avenue, Argonne, IL 60439 \\ ${ }^{2}$ Xi'an Jiao Tong University \\ 28 West Xian Ning Road, Xi'an, 710049, China \\ ${ }^{3}$ University of Illinois at Urbana-Champaign \\ 104 South Wright Street, Urbana, IL 61801
}

Abstract

Microbeam X-ray diffraction experiments were conducted at beam line 34-ID of the Advanced Photon Source (APS) on fission fragment energy Xe heavy ion irradiated single crystal Molybdenum (Mo). Lattice strain measurements were obtained with a depth resolution of $0.7 \mu \mathrm{m}$, which is critical in resolving the peculiar heterogeneity of irradiation damage associated with heavy ion irradiation. Q-space diffraction peak shift measurements were correlated with lattice strain induced by the ion irradiations. Transmission electron microscopy (TEM) characterizations were performed on the as-irradiated materials as well. Nanometer sized Xe bubble microstructures were observed via TEM. Molecular Dynamics (MD) simulations were performed to help interpret the lattice strain measurement results from the experiment. This study showed that the irradiation effects by fission fragment energy Xe ion irradiations can be collaboratively understood with the depth resolved X-ray diffraction and TEM measurements under the assistance of MD simulations. 


\section{INTRODUCTION}

Irradiation damage has for long been deemed a crucial problem due to its technical importance in nuclear fission and fusion reactors [1-3]. In the early stage of irradiation damage, individual defect structures such as dislocation loops and dislocations were observed to accumulate, causing the fundamental properties of the underlying material matrix to change. A classical example of radiation damage in $\mathrm{UO}_{2}$, which is the commonly used nuclear fuel material in water cooled fission reactors, was raised by Whapham et al [4]. Another well-known phenomenon that causes lattice strain to change in $\mathrm{UO}_{2}$ is often referred to as the Rim Effect (or grain refinement or recrystallization) at the very high burnup regime [5]. However, the underlying contributors to the lattice strain changes in such phenomenon are not yet well understood or well agreed upon. If one examines the measurement data in existing literatures like Hofman et al did in Ref [6], it becomes quite clear that there are not enough data in the burnup region between 0.3 and $10 \mathrm{GWd} / \mathrm{MTU}$ or in the displacement per atom (dpa) range of about 9 - $300 \mathrm{dpa}$.

Heavy ion irradiation has long been used as an alternative to neutron irradiation to study irradiation effects in nuclear materials [1]. Fission fragment induced irradiation damage is of great interest to nuclear fuel behaviors as it contributes to the majority of the damage done in the fuel materials. The formation and growth of fission gas bubbles in nuclear fuel materials and its implications are also of significant technical importance. The fuel swelling is strongly dependent on the evolution of fission gas bubble size and population. To this end, near fission fragment energy (104 MeV) Xe beam was used to investigate the effects of both irradiation damage and implanted Xe.

In this work, we attempted to characterize heavy ion beam damage of Xe ion (as the most abundant fission gas in nuclear fuels), with fission fragment energy, in single crystal Molybdenum (Mo) and the impact of such damage in terms of lattice strain changes. An irradiation damage dose range of 5-45 dpa was pursued. The X-ray diffraction study was performed at beam line 34 of the Advanced Photon Source (APS) at Argonne National Laboratory (ANL) with a microbeam diffraction technique. A differential-aperture X-ray microscopy (DAXM) technique was applied [7-9] in order to achieve $0.7 \mu \mathrm{m}$ depth resolution which is crucial to analyze irradiation induced strain in the material given the heterogeneity of the damage profile in the depth direction.

Transmission electron microscopy (TEM) investigations of the detailed microstructure were also performed. Focused Ion Beam (FIB) technique was used to generate lift-out specimens. In such a way, irradiation damage induced microstructure at different doses could be assessed. The results were shown to complement the microbeam 
diffraction measurements in the sense that they help clarify the contributions of both fission gas microstructures and defect microstructures to the overall strain change in the material.

In addition to the experimental techniques applied in this study, Molecular Dynamics (MD) simulations were performed to help provide modeling interpretations of measured lattice strain.

\section{EXPERIMENTAL}

\subsection{Specimen and heavy ion irradiation}

Mo was used in this work as a model material and single crystal was chosen so that grain boundary effects may be eliminated since no recrystallization is expected at this dose range at the temperature of irradiation [10]. Single crystal (99.999\% purity) Mo samples ([ $\left[\begin{array}{ll}0 & 1\end{array}\right]$ normal) were received from Good Fellow, Inc. The samples are 10mm in diameter and $2 \mathrm{~mm}$ in thickness. Mechanical polishing was performed to create a flat and smooth surface. The final finish of the polishing was by 1200 grit $\mathrm{SiC}$ paper. $104 \mathrm{MeV} \mathrm{Xe}^{26+}$ ion irradiation was conducted at the Argonne Tandem Linac Accelerator System (ATLAS). The ion beam was between $40^{\circ}$ and $45^{\circ}$ incident to the polished surface of the single crystal Mo samples $\left(45^{\circ}\right.$ angle was avoided to exclude the possibility of significant channeling). The use of this incident beam angle was partly due to the design of the sample stage to accommodate an Infra-red camera real time temperature monitoring system. The beam size was controlled to be about $12 \mathrm{~mm}$ in diameter which is slightly bigger than the size of the aperture $(9.5 \mathrm{~mm}$ in diameter). A beam current of $200 \mathrm{pnA}$ (particle nano-Ampere) was used which is equivalent to $1.25 \times 10^{12} \mathrm{Xe} / \mathrm{s}$. With a thermal couple connected to each sample stage and the Infra-red camera, the beam induced heating was shown to raise the temperature in the samples to about $380{ }^{\circ} \mathrm{C}$ at the center of the beam. At the periphery of the samples, the temperature was measured to be about $330^{\circ} \mathrm{C}$. Two single crystal Mo samples were irradiated. The final fluence of the irradiations were $1 \times 10^{16}$ and $2 \times 10^{16}$ $\mathrm{Xe} / \mathrm{cm}^{2}$ respectively. The fluence are equivalent to peak damages of about 22 and $44 \mathrm{dpa}$ according to the SRIM2008 calculation with a Kinchin-Pease model [11].

\subsection{Microbeam diffraction}

Differential Aperture X-ray Microdiffraction (DAXM) [7] experiment was carried out at beamline 34ID-E of the Advanced Photon Source. X-ray beam was focused to $0.5 \times 0.5 \mu \mathrm{m}^{2}$ in full-width half-maximum by a pair of Kirkpatrick-Baez mirrors. Sample was mounted in reflection geometry with incident angle of $45^{\circ}$, and diffraction signals were collected with a Perkin-Elmer XRD-1620 area detector. At selected Bragg reflection, energy of the x- 
ray beam was scanned to measure lattice d-spacing distributions. The differential aperture technique was applied to achieve spatial resolution along the x-ray penetration, which involves scanning a Pt wire over the sample surface at each step of the x-ray energy scan [9]. The combined energy-wire scan provides distribution of lattice d-spacing as a function of depth below the sample surface with a spatial resolution of $0.7 \mu \mathrm{m}$. Figure 1 shows a schematic of the $\mathrm{X}$ ray setup demonstrating the DAXM configuration. The energy of the beam was scanned over the energy range of $20.405 \mathrm{keV}-20.625 \mathrm{keV}$ for different samples with a step of $2.2 \mathrm{eV}$ for the $(0,0,4)$ or $(0,0,8)$ reflection which is the surface normal (error less than 0.5 degree according to Good Fellow, Inc.). The small X-ray beam size ( $0.5 \mu \mathrm{m})$ allowed non-destructive measurements of lattice strains in the mesoscale at different depths. Details describing applications of the DAXM technique can be found in Refs. [7-9, 12-16].

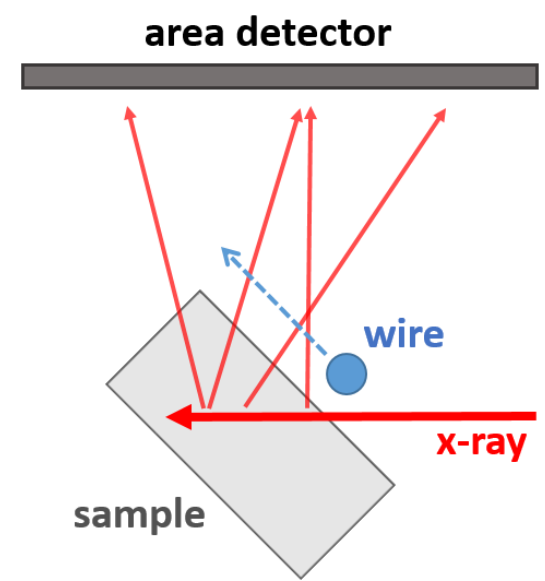

Figure 1. Schematic of the X-ray configuration of the DAXM technique.

The combined energy-wire DAXM scans were performed at different locations on each sample. The irradiation beam center on each sample was determined by high resolution Infra-red camera recorded thermal images. Locations were chosen at $0.9 \mathrm{~mm}, 1.8 \mathrm{~mm}$, and $2.7 \mathrm{~mm}$ away radially from the beam center and thereby a measurement center line was determined on each sample. According to the measured distribution of the beam current, which is close to a Gaussian distribution, the peak damage at each location can be estimated by SRIM simulation. The resulting peak damages in terms of dpa are reported in Table 1.

Table 1. SRIM calculated peak damage in terms of dpa at each location on the two samples.

\begin{tabular}{|c|c|c|}
\hline Location & $\begin{array}{c}\text { Peak damage at the location (dpa, } \\
\text { sample with peak damage 22 dpa) }\end{array}$ & $\begin{array}{c}\text { Peak damage at the location (dpa, } \\
\text { sample with peak damage 44 dpa) }\end{array}$ \\
\hline beam center & 22 & 44 \\
\hline $0.9 \mathrm{~mm}$ away from beam center & 18.9 & 37.8 \\
\hline $1.8 \mathrm{~mm}$ away from beam center & 11.9 & 23.8 \\
\hline $2.7 \mathrm{~mm}$ away from beam center & 5.5 & 11 \\
\hline
\end{tabular}




\subsection{Transmission electron microscopy}

Upon finish of the microbeam diffraction measurements, FIB technique was used to extract thin TEM specimens from different locations along the diffraction measurement center line in order to probe the microstructures at different irradiation dose regions. The TEM specimens were prepared by an FEI Helios 600 i FIB. The samples were cut, lifted out, and thinned by a $30 \mathrm{keV} \mathrm{Ga}$ ion beam. The radiation damage due to the $30 \mathrm{keV} \mathrm{Ga}$ ions was then eliminated by cleaning the specimens using a $2 \mathrm{keV}$ Ga ion beam. The FIB preparation procedure usually produces a $10 \mu \mathrm{m} \times 10 \mu \mathrm{m}$ electron transparent region appropriate for TEM examination.

\section{MODELING AND SIMULATION}

In order to help interpret the lattice strain measurement results by microbeam diffraction, MD simulations were performed. The interatomic potential used in this work was developed by Smirnova et al [17]. This embedded atom method potential was fitted using a force-matching method and a data-set of density functional theory (DFT) calculated atomic forces. The MD simulations were performed by LAMMPS (Large-scale Atomic Molecular Massively Parallel Simulator) [18]. To study the effect of Xe on the lattice strain of Mo matrix, two scenarios of Xe ions in Mo matrix were considered: (1) Xe gas occupies the Mo lattice or interstitial site and forms solution atoms; (2) Xe gas atoms form bubbles in Mo. In the first scenario, the MD simulations were performed in a system containing about 16000 atoms $(20 \times 20 \times 20$ conventional bcc unit cells) with three different Xe concentrations, i.e., $0.3 \%, 0.5 \%$, and $0.8 \%$. Our study shows that the formation energy of Xe substitution at Mo lattice site is much lower than that of Xe occupying an interstitial site in Mo. Therefore, most of Xe atoms were introduced as substitution defects and less than $20 \%$ Xe atoms were placed at the octahedral interstitial sites of Mo. In the second scenario, a much larger system containing about 432000 atoms $(60 \times 60 \times 60$ conventional bcc unit cells $)$ was used to accommodate nano-sized Xe bubbles. Two different Xe concentrations, i.e., $0.5 \%$, and $0.8 \%$, were studied by MD calculations. In this case, most of the Xe gas atoms were introduced in the gas bubbles and less than $6 \% \mathrm{Xe}$ atoms were placed at the octahedral interstitial sites of Mo. Examples of Mo structures with $0.5 \%$ Xe as solution atoms or forming gas bubbles are shown in Fig. 2. The simulation boxes were equilibrated at $300 \mathrm{~K}$ in an isothermal-isobaric (NPT) ensemble with atmospheric pressure up to $1 \mathrm{~ns}$. The equilibrium lattice parameter of Mo with Xe atoms at the end of simulations was used to estimate the lattice strain due to incorporation of Xe. 


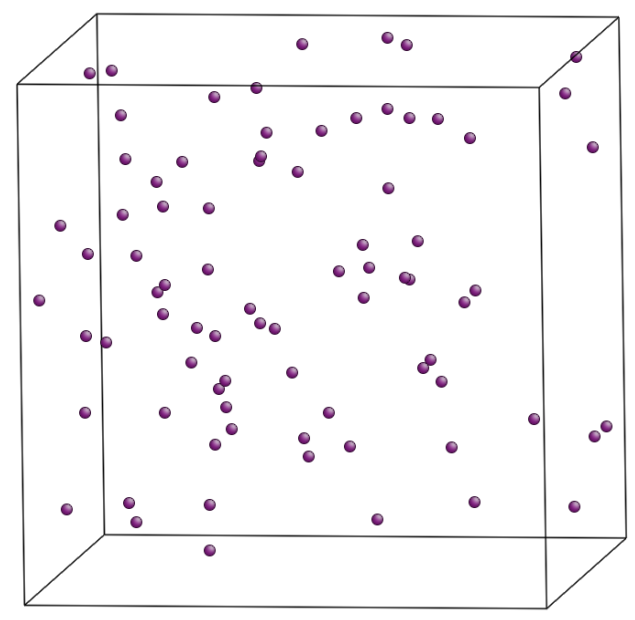

(a)

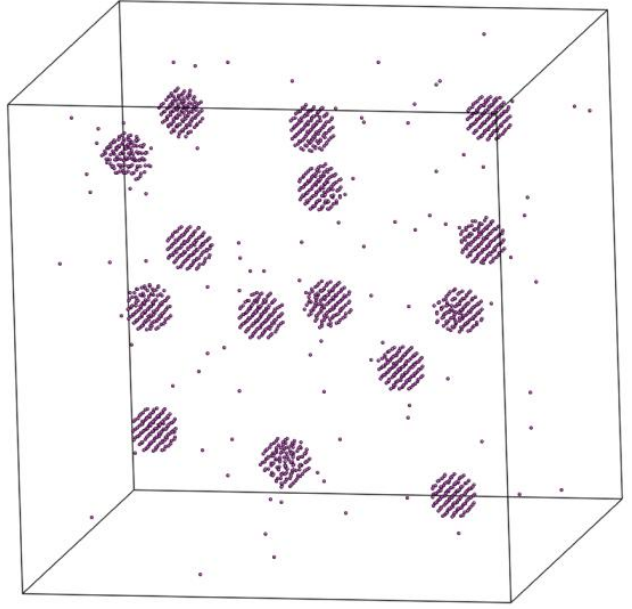

(b)

Figure 2. Atomic structures of bcc Mo irradiated with about $0.5 \%$ Xe atoms: (a) Xe gas atoms form solution in Mo matrix and (b) Xe gas atoms form bubbles in Mo matrix (b). For easy visualization, only Xe atoms are shown in the pictures.

\section{RESULTS AND DISCUSSION}

4.1 Q-depth map and lattice strain

Fig. 3 (a) shows the 2-dimensional (2-D) Q-depth map of x-ray diffraction intensities for un-irradiated single crystal Mo, where depth denotes the direction of $\mathrm{x}$-ray penetration, while Q stands for the magnitude of the $\mathrm{X}$-ray scattering vector and is equivalent to $2 \pi / d$, with $d$ being the lattice $d$-spacing of the diffracting atomic planes. It needs to be noted that the depth values here are arbitrary and should be viewed as a relative measure. As expected, the peaks in Q-space in the Q-depth map lines up horizontally (represented by the black line) indicating that there is no variation on the internal strain across different depths. The darkest part at each depth of the Q-depth map marks the peak of the Q distribution at that particular depth. As an example, Fig. 3 (b) shows, at a particular depth, the Q peak and its corresponding fit with a Lorentzian peak function. The dotted vertical line indicates where the nominal $\mathrm{Q}$ value of Mo should be. From the difference between the fitted Q peak center and the nominal Q value, $\Delta \mathrm{Q} / \mathrm{Q}$ can be calculated which is the same as $\Delta \mathrm{d} / \mathrm{d}$. Therefore, lattice strain becomes a direct result of fitting to the measured $\mathrm{Q}$ peak. It can be seen from Fig. 3 (b) that the fitted peak center in terms of Q value is slightly higher than the nominal Q value for Mo indicating a compressive strain of $-2.9 \times 10^{-5}$. It should be noted that such a small strain is within the measurement uncertainty of about $\pm 5 \times 10^{-5}$ confirming the unstrained nature of the un-irradiated single crystal Mo. 


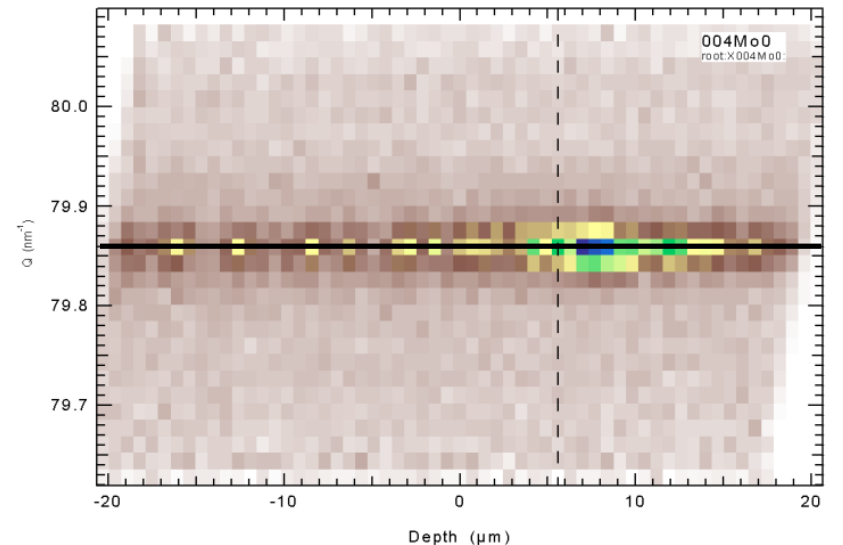

(a)

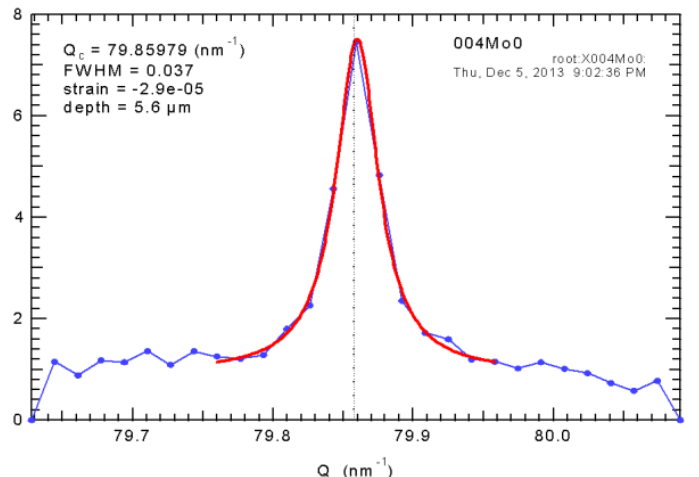

(b)

Figure 3. On the left (a) Q-depth map of un-irradiated single crystal Mo and on the right, (b) Q peak fitting by a

\section{Lorentzian peak function}

In addition to analyzing the Q-depth map of the un-irradiated single crystal Mo specimen, similar Q-depth maps of the two irradiated specimens measured at various locations (i.e. beam center, $0.9 \mathrm{~mm}, 1.8 \mathrm{~mm}$ and $2.7 \mathrm{~mm}$ away from the beam center along the measurement center line) have been analyzed. It is, however, impractical to show all Q-depth maps. Consequently, only one additional Q-depth map taken $2.7 \mathrm{~mm}$ away from the beam center on the 22 peak dpa specimen is shown in Fig. 4 as an example of the Q-depth map of irradiated specimen. Associated peak fittings at several depths are also demonstrated. Again, $(0,0,4)$ reflection, which is the normal direction of the specimen, was used for the diffraction measurement. The dotted horizontal black line in the Q-depth map marks the nominal Q value of Mo, which is coincident with the long tail of the diffraction signal into the depth beyond $20 \mu \mathrm{m}$. It is then meaningful to compare the $\mathrm{Q}$ value of the anticipated irradiated region (through SRIM simulation) to that of the un-irradiated region. It can be seen that for the first 6-7 $\mu \mathrm{m}$, the lattice in the sample normal direction is under tension as $\mathrm{Q}$ value is smaller than that of the un-irradiated region. In fact, the maximum tensile strain in the sample is about $5.1 \times 10^{-4}$ relative to the un-irradiated region. This is a typical case that is representative of all measured Qdepth maps. 


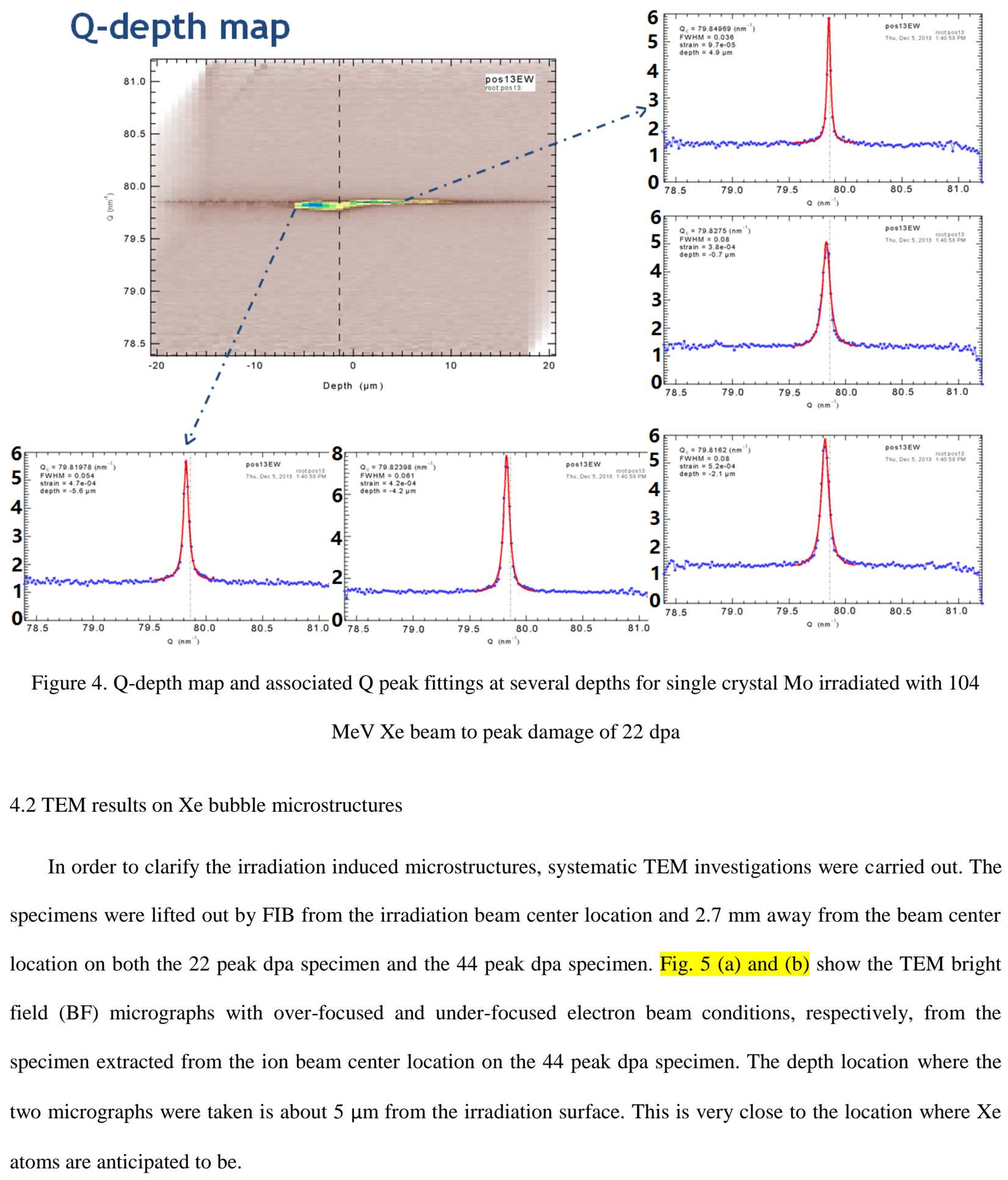




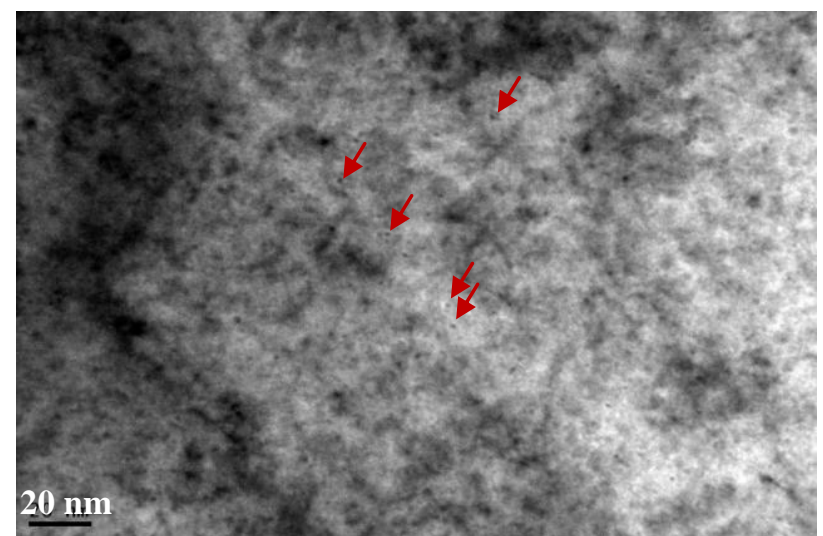

(a)

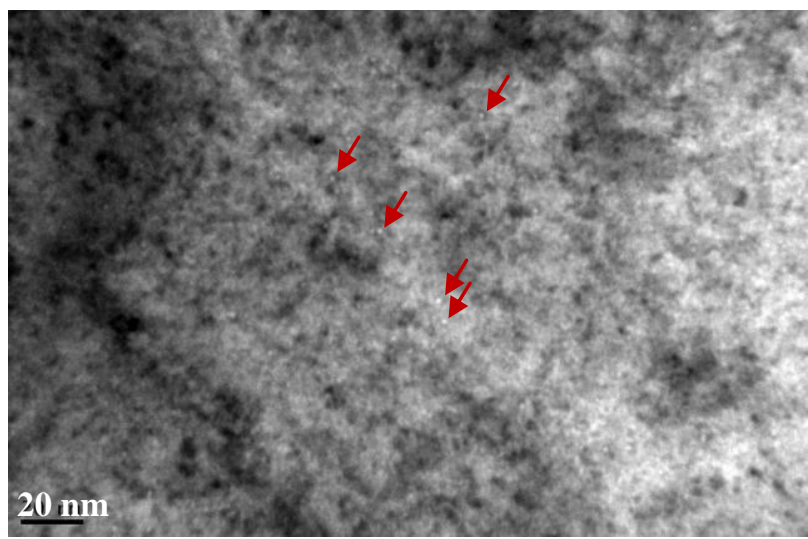

(b)

Figure 5. TEM BF micrographs with (a) over-focused and (b) under-focused electron beam conditions, respectively,

from the specimen extracted from the ion beam center location on the 44 peak dpa specimen. Red arrows mark where Xe bubbles are located.

Xe bubbles can be clearly seen by the inversed-focus-contrast such that under over-focused electron beam condition they would appear as black dots whereas under under-focused electron beam condition they would appear as white dots. To assist with locating the Xe bubbles, several red arrows are placed on a few Xe bubbles to point out some examples. It should be noted that Xe bubbles were only found in the 44 peak dpa specimen and the beam center location in the 22 peak dpa specimen indicating that there exists a solubility limit above which Xe would precipitate out in form of bubbles. Below such solubility limit, Xe should reside in form of atoms in solution. However, the possibility of gas bubbles with sizes below the TEM detection threshold needs to be considered as well. For the TEM used in this work, the resolution limit is about $0.6 \mathrm{~nm}$. If a more conservative estimate of the resolution limit is taken, one could assume that $0.8 \mathrm{~nm}$ diameter bubbles can be detected by the TEM. Such size translates to only 5-6 Xe atoms residing in the bubble even if over-pressurized solid state bubbles are assumed. Therefore, if there were bubbles below TEM detection threshold, these bubbles should unanimously contain less than $6 \mathrm{Xe}$ atoms each. The Xe bubbles observed have an average size of $2.5 \mathrm{~nm}$ in diameter and the variation in bubble size is relatively small (the smallest observed bubble has a diameter of about $2.2 \mathrm{~nm}$ ). Thus, by estimate assuming highly pressurized bubbles, the number of Xe atoms in a bubble is over 100 even for the smallest bubble. It is quite difficult to picture a scenario where the size of gas bubbles have such distinguished bi-modal distribution (one size class of less than 6 atoms and the other more than 100 atoms). Consequently, it is believed that the $\mathrm{Xe}$ atoms not residing in observable bubbles should be in solid solution with the Mo matrix.

4.3 MD simulations of Xe contribution to lattice strain 
To assess the contributions to lattice strain by $\mathrm{Xe}$ in solid solution and by $\mathrm{Xe}$ in form of bubbles, MD simulations become very useful. With the current interatomic potential, the lattice constant of pure bcc Mo was predicted to be $3.151 \AA$, which is a little larger than that measured by experiment, i.e. 3.147 $\AA$. The overestimation of the lattice constant of bcc Mo is due to the fact that the EAM potential of U-Mo-Xe was fitted from DFT calculations of Mo using the generalize gradient approximation (GGA). It is known that GGA approximation always overestimates the lattice constants of metals [19]. Since we are interested in the lattice change due to Xe gas, such overestimation won't affect the following study of lattice strain. The averaged lattice strain $\varepsilon_{\text {ave }}$ due to introducing Xe gas atoms was estimated by

$$
\varepsilon_{\text {ave }}=\frac{a_{M o X e_{x}}-a_{M o}}{a_{M o}},
$$

where $\mathrm{a}_{\mathrm{MoXex}}$ is the averaged lattice constant of Mo with Xe atoms, and $\mathrm{a}_{\mathrm{Mo}}$ the lattice constant of pure bcc Mo. Fig. 6 shows the predicted Xe concentration dependence of the lattice strain of Mo irradiated with Xe. In both cases, the lattice strains follow a linear dependence on the Xe concentration. However, the lattice strain with Xe forming gas bubbles is noticeably higher than that with Xe as solution atoms. This can be understood by the difference of surface pressure exerted by the Mo matrix on the Xe bubble. The surface pressure $\mathrm{P}$ due to curvature can be approximated by

$$
P=\frac{2 \gamma_{\mathrm{int}}}{r}
$$

where $\gamma_{\mathrm{int}}$ is the interfacial energy between Mo matrix and Xe bubble and $\mathrm{r}$ the radius of gas bubble. When Xe forms solution atoms in the matrix of Mo, it can be assumed as the extreme case of gas bubble with only one atom. Therefore, the gas pressure in the bubble decreases as the bubble size increases thereby increasing the volume per Xe atom. This eventually leads to larger strain in the matrix of Mo. 


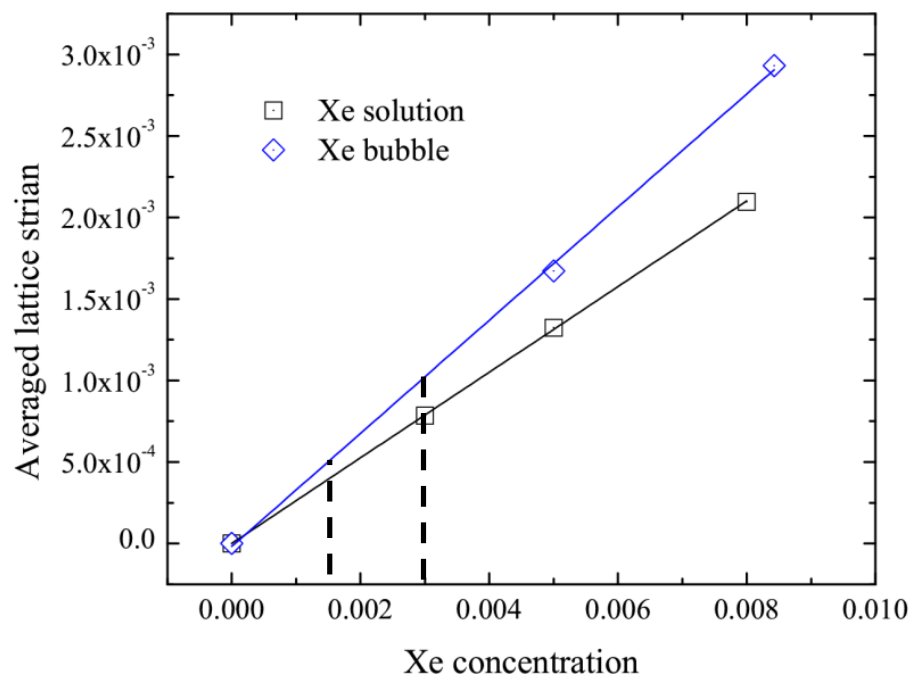

Figure 6. Xe concentration dependence of the averaged lattice strain in bcc Mo with Xe gas forming solution atoms or gas bubbles. The solid lines from linear fitting are guides to the eyes.

\subsection{Analyses of lattice strain measurements}

It is imperative to realize that the lattice strains obtained from the microbeam diffraction measurements are extremely complex to analyze as the lattice strains consists of many components, i.e. contributions from different irradiation induced microstructures. In general, the lattice strain can be dissected into three components via the following equation:

$$
\varepsilon=\varepsilon_{X e}+\varepsilon_{X e \text { bubble }}+\varepsilon_{\text {defects }},
$$

where $E$ is the lattice strain, in this case, along the $\left[\begin{array}{lll}0 & 0 & 1\end{array}\right]$ direction; $\varepsilon_{\mathrm{Xe}}$ is the contribution by Xe in solid solution; $\varepsilon_{\mathrm{Xe}}$ bubble is the contribution by Xe in form of bubbles and $\varepsilon_{\text {defects }}$ is the contribution by defects generated by the heavy ion irradiation.

Fig. 7 shows depth-resolved $\left[\begin{array}{lll}0 & 0 & 1\end{array}\right]$ lattice strain measurements at different locations in the 22 dpa peak damage specimen. It can be seen that lattice strain generally increases to reach the maximum strain at around 4-5 $\mu \mathrm{m}$ depths. This is an anticipated result as both irradiation induced damage and Xe atoms are concentrated in this region. However, the comparison between the overall strain profiles is somewhat unexpected particularly because the measured lattice strains are much higher at a location that received the least irradiation damage (the $2.7 \mathrm{~mm}$ away from beam center location). In order to keep the figure as clean as possible, error bars are omitted from the figure. 
The uncertainty of the measurements through DAXM technique is about $\pm 5 \times 10^{-5}$ [20]. Clearly, at depths less than 6 $\mu \mathrm{m}$ from the irradiation surface, the measured lattice strains at $2.7 \mathrm{~mm}$ away from the beam center are significantly higher than those measured from any other locations even when errors are taken into account. As the strain contributions by Xe gas atoms either in solution or in form of bubbles increase monotonically with Xe concentration (and therefore irradiation damage), the overall strain difference has to be explained by the contributions from the irradiation induced defects. In order to clarify this, two horizontal lines (black and pink) are placed on the figure at the magnitudes where anticipated $\left[\begin{array}{lll}0 & 0 & 1\end{array}\right]$ lattice strain contributions from Xe are estimated via MD simulations. It should be realized that due to the isotropic nature of the straining by Xe in solution and Xe bubbles, the averaged lattice strain represents the lattice strain in the [lll $\left.0 \begin{array}{ll}0 & 1\end{array}\right]$ direction. At this irradiation dose (22 peak dpa), Xe bubbles were not observed at the peak damage location indicating that Xe atoms should be in solid solution with the Mo matrix. Consequently, strain contribution by Xe in solution was used for the two horizontal lines (Xe concentration was estimated to be 1.5 at.\% at 22 dpa peak damage location according to SRIM simulation). It should be noted that the average Xe concentration over the depth region covered by the green bar was used to estimate the Xe contribution to the lattice strain. The black arrows represent the contribution to the lattice strain from irradiation induced defects for the locations of irradiation beam center and $2.7 \mathrm{~mm}$ away from the beam center. Strain contributions from irradiation induced defects were calculated by deducing the estimated strain contribution by Xe (including Xe in solution and Xe in bubbles if bubbles exist) from the total measured strain. It is then observed that the defect contribution to the lattice strain at the location of $2.7 \mathrm{~mm}$ away from the beam center is as much as $4.7 \times 10^{-4}$ and the defect contribution to the lattice strain at the location of the irradiation beam center is in fact negative (about $-0.8 \times 10^{-4}$ ). The situation for the 44 dpa peak damage specimen (Fig. 8) is somewhat similar in the sense that the contribution to the lattice strain by defects at the location $2.7 \mathrm{~mm}$ away from the beam center on this specimen is positive (about $3.2 \times 10^{-4}$ ) but the contribution by defects at the beam center is negative (about $-5.7 \times 10^{-4}$ ). At the irradiation dose of 44 peak dpa, Xe bubbles were observed at the peak damage location (see Fig. 5). Via calculations assuming near solid state Xe, the portion of Xe residing in bubbles was estimated to be roughly $20 \%$ of all implanted Xe. Consequently, strain contribution was partitioned into $20 \%$ by Xe in bubbles and $80 \%$ by Xe in solution. The resulting strain value was then used for the the horizontal line for the center location (black line, Xe concentration was estimated to be 3 at. $\%$ at 44 dpa peak damage location according to SRIM simulation). For the location $2.7 \mathrm{~mm}$ from the beam center, no Xe bubbles are expected to form (as no Xe bubbles were observed in the 
higher damage location with 22 peak dpa damage). Therefore strain contribution by Xe in solution was used for the corresponding horizontal line (pink line).

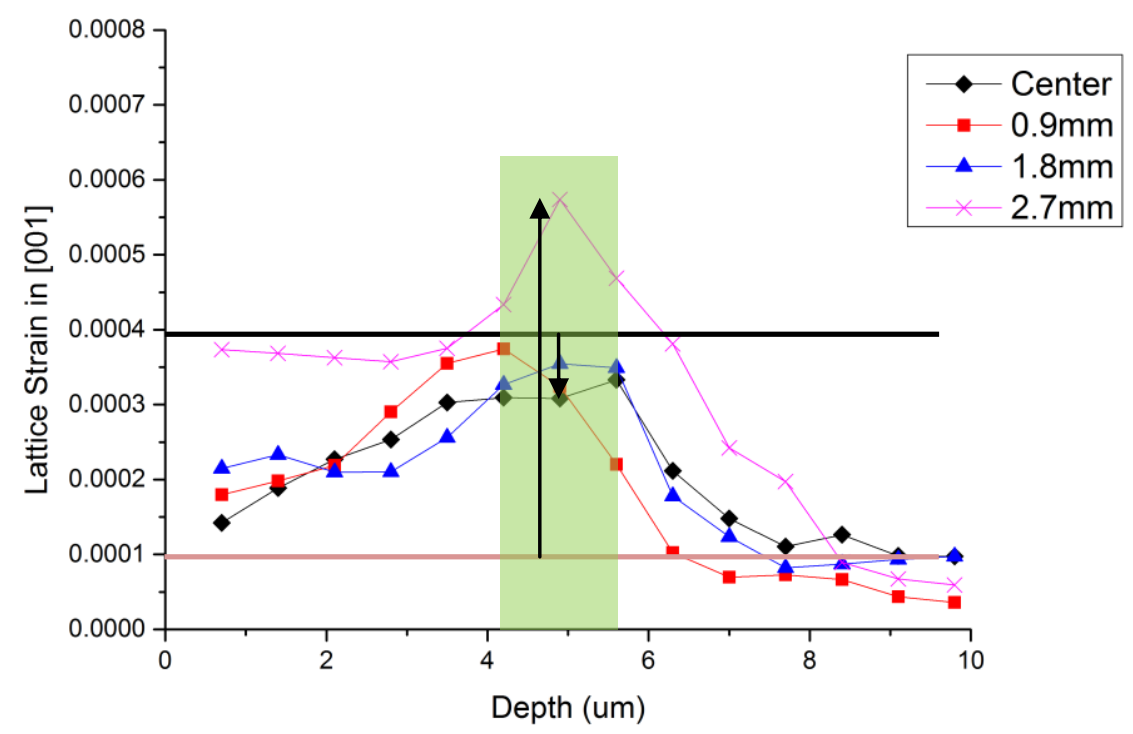

Figure 7. Depth-resolved $\left[\begin{array}{lll}0 & 0 & 1\end{array}\right]$ lattice strain measurements at different locations in the 22 dpa peak damage specimen. Two horizontal lines (light blue and green) indicate the magnitudes where anticipated [ $\left[\begin{array}{llll}0 & 0 & 1\end{array}\right]$ lattice strain contributions from Xe are estimated via MD simulations. The black arrows represent the contribution to the lattice strain from irradiation induced defects.

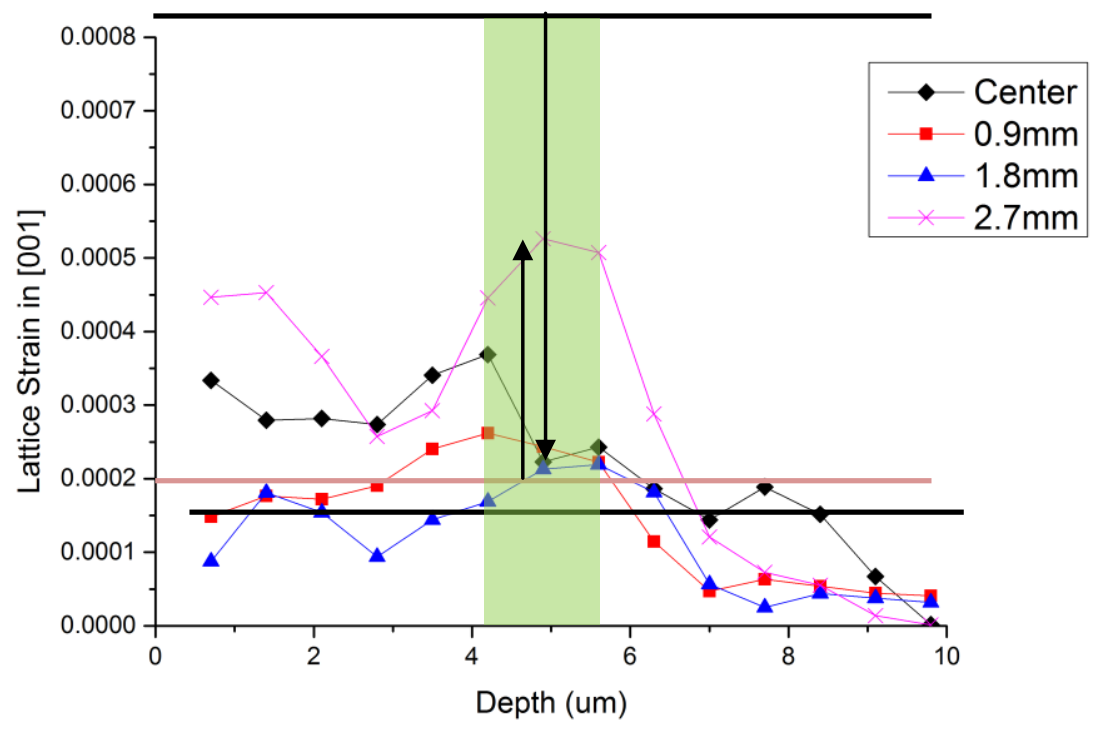


Figure 8. Depth-resolved [ $\left[\begin{array}{lll}0 & 0 & 1\end{array}\right]$ lattice strain measurements at different locations in the 44 dpa peak damage specimen. Two horizontal lines (light blue and green) indicate the magnitudes where anticipated [ $\left[\begin{array}{lllllllll}0 & 0 & 1\end{array}\right]$ latice strain contributions from Xe are estimated via MD simulations. The black arrows represent the contribution to the lattice strain from irradiation induced defects.

In any case, irradiation induced point defects and defect clusters such as Frenkel pairs, interstitial dislocation loops and dislocations should not introduce compressive strain components to the material matrix. A plausible explanation would be that the Xe bubble induced strain is relaxed by the irradiation induced defects. In fact, a higher number density of Xe bubble microstructures has been seen in the FIBed TEM specimen from the irradiation beam center location of the 44 dpa peak damage specimen than from the irradiation beam center location of the $22 \mathrm{dpa}$ peak damage specimen. This could explain why there is a larger negative contribution by defects associated with the 44 dpa peak damage specimen at the beam center location. Another possibility is that vacancy dislocation loops may have contributed to the decrease in lattice strain. Earlier researchers have argued that vacancy loops have been observed in irradiated $\mathrm{UO}_{2}$ albeit the conclusion was based on logical deduction, not on direct characterization [4].

Fig. 9 plots measured [ $\left[\begin{array}{lll}0 & 0 & 1\end{array}\right]$ peak lattice strain versus irradiation damage in terms of peak dpa. Peak lattice strain refers to the maximum lattice strain along the depth direction. It is perceivable that lattice strain decreases with increasing irradiation damage. It is then worthwhile to investigate what microstructural components might have contributed to this decrease in lattice strain.

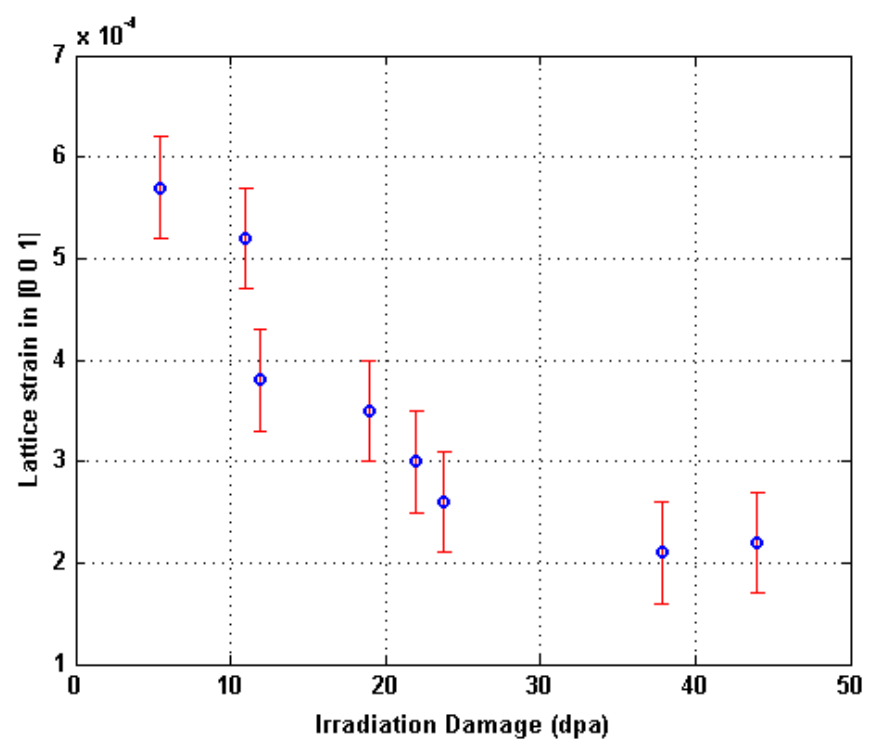

Figure 9. Measured [ $\left[\begin{array}{lll}0 & 0 & 1\end{array}\right]$ peak lattice strain versus irradiation damage in terms of peak dpa. 
To this end, further TEM investigations were carried out at the locations where, according to SRIM calculations, 5.5 and 11 dpa of damage were received. Fig. 10 (a) and (b) show the bright field TEM micrographs representing the microstructures of these locations. Despite the slight difference in magnification, it is easily seen that the defect microstructures are significantly different. At the location where 5.5 dpa irradiation damage is received, there exists a high number density of individual dislocation loops whose nature has not been determined in this work. However, it's very likely that these loops are of interstitial nature according to Li et al and Xu et al [2123]. At the higher irradiation damage location where $11 \mathrm{dpa}$ of damage is received, many dislocation microstructures can be observed. These dislocations are thought to be produced through the coalescence or collapse of individual dislocation loops. It can also be seen that there still exists individual dislocation loops in between these dislocation structures. According to Hofman et al [6], production of interstitial dislocation loops tend to increase lattice parameter but lattice parameter decreases when dislocation loops evolve into a dislocation network. This could then explain the decrease of lattice strain towards higher damage in terms of dpa. At $44 \mathrm{dpa}$, the contrast (in Fig. 5 (a) and (b)) becomes too complex to resolve individual dislocations and dislocation loops or even a dislocation network. This is quite typical of TEM observations of materials with high dose of irradiation damage.

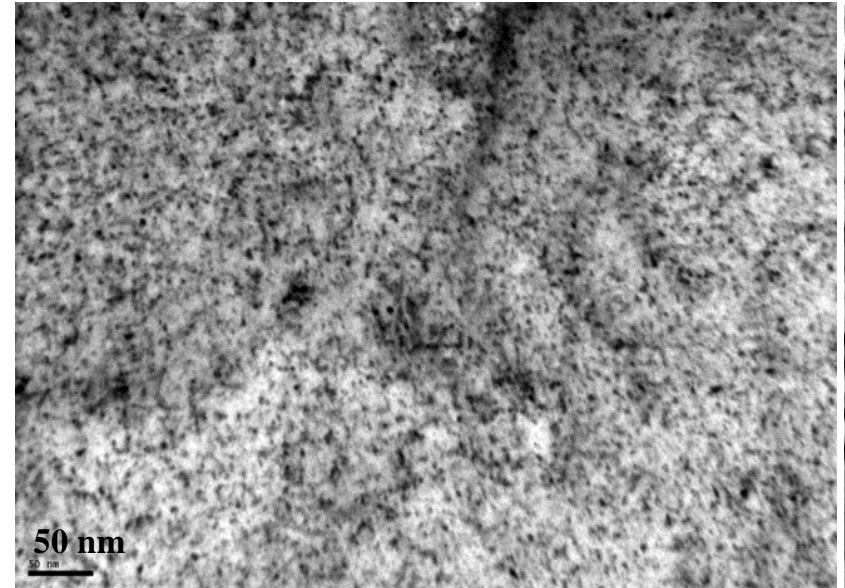

(a)

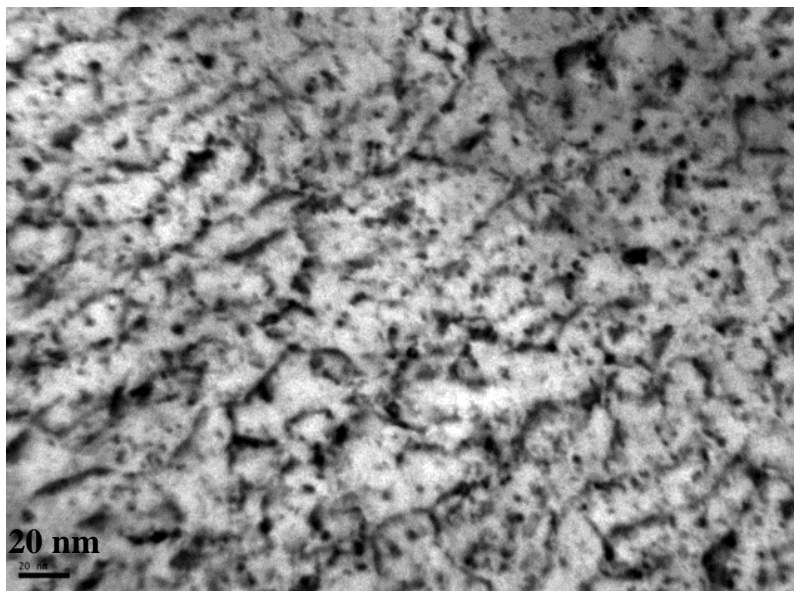

(b)

Figure 10. Bright field TEM micrograph of defect microstructures at different locations: (a) 5.5 dpa location and (b)

11 dpa location.

\section{CONCLUSIONS}

Microbeam X-ray diffraction experiments were conducted at beam line 34-ID of the APS on fission fragment energy Xe heavy ion irradiated single crystal Mo. Lattice strain measurements were obtained with a depth resolution of $0.7 \mu \mathrm{m}$, which is critical in resolving the peculiar heterogeneity of irradiation damage associated with heavy ion 
irradiation. From the measurements, it is observed that lattice strain decreases with increasing irradiation damage. Through TEM characterizations that were performed on the as-irradiated materials, high density of dislocation loops was found at the location where 5.5 dpa irradiation damage was received whereas dislocations were found at the location where $11 \mathrm{dpa}$ irradiation damage was received. The evolution from individual dislocation loops to dislocations and then to a dislocation network is thought to lower the lattice strain. In addition, nanometer sized Xe bubble microstructures were observed via TEM. MD simulations were also performed to help interpret the lattice strain measurement results from the experiment. It was shown that tensile lattice strain contributions by both Xe atoms in solution and Xe bubbles increase linearly with Xe concentration. When Xe contributions to the $\left[\begin{array}{lll}0 & 0 & 1\end{array}\right]$ lattice strain are accounted for by partitioning MD calculated Xe contributions of the lattice strain to the measured lattice strain, it was thought that defects may have contributed to the relaxation of lattice strain induced by Xe bubble microstructures.

\section{ACKNOWLEDGMENTS}

The authors would like to thank the help of Dr. Shaofei Zhu and Matthew Hendricks on the ATLAS irradiations. This research used resources of ANL's ATLAS facility, which is a DOE Office of Science User Facility. This work was supported under US Department of Energy Contract DE-AC02-06CH11357. Use of the Center for Nanoscale Materials, an Office of Science user facility, was supported by the U. S. Department of Energy, Office of Science, Office of Basic Energy Sciences, under Contract No. DE-AC02-06CH11357. We gratefully acknowledge the computing resources provided on Blues, a high-performance computing cluster operated by the Laboratory Computing Resource Center at Argonne National Laboratory.

\section{REFERENCES}

[1] G. Was, "Fundamentals of Radiation Materials Science: Metals and Alloys," Springer, July, 2007.

[2] D. R. Olander, "Fundamental Aspects of Nuclear Reactor Fuel Elements," Technical Information Center, Energy Research and Development Administration, June, 1976.

[3] K. L. Murty, I. Charit, “An Introduction to Nuclear Materials,” Wiley VCH, January, 2013.

[4] A. D. Whapham, B. E. Sheldon, Philosophical Magazine, 12:120, 1179, 1965.

[5] J. Spino, D. Papaioannou, Journal of Nuclear Materials, 281, 146, 2000.

[6] G. L. Hofman, Y. S. Kim, Nuclear Engineering and Technology, 37, 299, 2005. 
[7] B. C. Larson, W. Yang, G. E. Ice, J. D. Budai, J. Z. Tischler, Nature, 415, 887, 2002.

[8] G. E. Ice, R. I. Barabash, F. Walker, Composites Part B: Engineering, 36, 271, 2005.

[9] W. Yang, B. C. Larson, G. E. Ice, J. Z. Tischler, J. D. Budai, K. S. Chung, Applied Physics Letter, 82, 3856, 2003.

[10] D. Yun, M. A. Kirk, P. M. Baldo, J. Rest, A. M Yacout, Z. Z. Insepov, Journal of Nuclear Materials, 437, $240,2013$.

[11] J. Ziegler, SRIM, 2010. 〈http://www.srim.org>

[12] O. M. Barabash, M. Santella, R. I. Barabash, G. E. Ice, J. Z. Tischler, Journal of Materials, 62, 29, 2010.

[13] H. Bei, R. I. Barabash, G. E. Ice, W. Liu, J. Z. Tischler, E. P. George, Applied Physics Letter, 93, 071904, 2008.

[14] R. I. Barabash, H. Bei, Y. F. Gao, G. E. Ice, E. P. George, Journal of Materials Research, 25, 2, 2010.

[15] R. I. Barabash, H. Bei, Y. F. Gao, G. E. Ice, Acta Materialia, 58, 6784, 2010.

[16] R. I. Barabash, H. Bei, G. E. Ice, Y. F. Gao, O. M. Barabash, Journal of Metallurgy, 63, 30, 2011.

[17] D. E. Smirnova, A. Y. Kuksin, S. V. Starikov, V. V. Stegailov, Z. Insepov, J. Rest, and A. M. Yacout, Modelling and Simulation in Materials Science and Engineering, 21, 035011, 2013.

[18] S. Plimpton, Journal of Computational Physics, 117, 1, 1995.

[19] S. L. Shang, A. Saengdeejing, Z. G. Mei, D. E. Kim, H. Zhang, S. Ganeshan, Y. Wang, and Z. K. Liu, Computational Materials Science, 48, 813, 2010.

[20] Private communications with B. C. Larson.

[21] M. Li, N. Hashimoto, T. S. Byun, L. L. Snead, S. J. Zinkle, Journal of Nuclear Materials, 367-370, 817, 2007.

[22] M. Li, M. Eldrup, T.S. Byun, N. Hashimoto, L. L. Snead, S. J. Zinkle, Journal of Nuclear Materials, 376, $11,2008$.

[23] D.H. Xu, B.D. Wirth, M.M. Li, M.A. Kirk, Acta Materialia, 60, 4286, 2012. 